\title{
Incerteza na Medição dos Parâmetros Geométricos do Cordão de Solda
}

\author{
(Measurement Uncertainty of Geometric Parameters in Weld Beads)
}

\author{
Rosenda Valdés Arencibial ${ }^{1}$ Eduardo Manuel Díaz Cedré2, Amado Cruz Crespo ${ }^{3}$, Antonio Piratelli-Filho ${ }^{4}$ \\ ${ }^{1}$ Universidade Federal de Uberlândia - FEMEC. Av. João Naves de Ávila, 2121 - Bloco 1M, Uberlândia-MG, \\ e-mail: arvaldes@mecanica.ufu.br ${ }^{2,3}$ Centro de Investigaciones de la Soldadura, Universidad Central "Marta Abreu” de las Villas, \\ Carretera a Camajuaní, km 51/2, Santa Clara, Villa Clara, Cuba. e-mail: cedre@.uclv.edu.cue acruz@uclv.edu.cu \\ ${ }^{4}$ Universidade de Brasília (UnB) - Faculdade de Tecnologia ( FT). Depto. Engenharia Mecânica - Campus Universitário Darcy \\ Ribeiro.ASA NORTE.Brasília -DF - Brasil.e-mail: pirateli@unb.br
}

\begin{abstract}
Resumo
Este trabalho apresenta uma metodologia para estimar a incerteza associada à medição dos parâmetros que definem a geometria do cordão de solda, especificamente da área do cordão, de forma a atender à exigência estabelecida pela norma NBR ISO/IEC 17025. A qualidade geométrica dos corpos de prova utilizados durante as medições foi ainda avaliada através da medição dos desvios geométricos de planeza e de perpendicularidade. As seguintes etapas foram propostas e realizadas: identificação dos parâmetros que definem a geometria do cordão de solda; identificação e estudo das variáveis que afetam a medição destes parâmetros; adoção do modelo matemático para estimativa da incerteza de cada parâmetro; planejamento e execução dos experimentos para o levantamento dos dados, cálculo da incerteza e, finalmente, análise e discussão dos resultados. Através da análise dos resultados foi possivel concluir que as incertezas provenientes da calibração do sistema de medição e relativa ao desvio de perpendicularidade contribuíram significativamente para a incerteza final. As análises despertaram uma preocupação com relação aos valores permissiveis para o desvio de perpendicularidade dos corpos de prova utilizados durante as medições.
\end{abstract}

Palavras-Chave: Soldagem, geometria do cordão de solda, incerteza de medição, planeza, perpendicularidade.

Abstract: This work presents a methodology to estimate the uncertainty associated to the measurement of the weld bead geometric parameters in order to address the requirements of NBR ISO/IEC 17025 Standard. The specimen geometric quality was additionally evaluated through the measurement of flatness and perpendicularity deviations. The following steps were proposed and executed: identification of weld bead geometric parameters; identification and study of the variables that affect measurement of the identified parameters; adoption of a mathematical model to estimate the uncertainty for each parameter; planning and execution of the experiments for data obtaining; uncertainty determination, analysis and discussion of the results. It was concluded that the most important variables influencing the final uncertainty were the uncertainty associated to the measuring system calibration and the perpendicularity deviation. The analysis warned about the permissible values of perpendicularity deviations of specimens used during the measurements.

Key-words: Welding, weld bead geometry, uncertainty, flatness, perpendicularity.

\section{Introdução}

A geometria dos cordões de solda constitui um elemento de primeira importância quando se avalia a qualidade da união soldada. A literatura da área apresenta pesquisas onde a geometria dos cordões constitui aspecto essencial do enfoque [14]. Outros trabalhos, mais abrangentes, consideram a geometria do cordão como um elemento inevitável na caracterização [5, 6]. Ainda, um grupo de pesquisadores utiliza a geometria como critério quantitativo para a predição de parâmetros de processo, realçando a sua importância [7-11].

(Recebido em 19/05/2010; Texto final em 30/03/2011).
Para o dimensionamento de qualquer união soldada, diversos aspectos devem ser considerados, tais como: o nível de solicitação dos esforços, o processo de fixação, o grau de dificuldade da operação, a proporção geométrica entre cordão e componentes soldados, a produção necessária, a composição do material de base, o grau de automatização do processo, além, do custo envolvido.

Contudo, um adequado dimensionamento não é suficiente para garantir a qualidade do produto final dos processos de soldagem, sendo indispensável o desenvolvimento de meios e métodos que permitam a verificação das dimensões a fim de comprovar se estão de acordo com o especificado na fase de projeto. Assim sendo, a medição dos parâmetros geométricos dos cordões deve ser executada de forma cuidadosa para garantir a obtenção de resultados confiáveis. Os resultados das medições dos parâmetros penetração, largura, reforço, área de penetração 
e área de reforço devem ser apresentados em conjunto com os valores de incerteza associados, para que estejam de acordo com as normas em vigor.

Para medir os parâmetros que definem a geometria do cordão de solda, sistemas de captação de imagens associados a programas computacionais são utilizados com frequência. Neste caso, a medida básica é o número de pixels ocupados pelo mensurando. A aplicação desta técnica, utilizando fotografias, facilitou muito as medições dos parâmetros que definem a geometria do cordão, porém, aumentou a complexidade do cálculo da incerteza. Também, elevou o nível de exigências quanto à capacitação do pessoal que realiza a medição, pois, além do ajuste necessário do microscópio, o sistema deve ser calibrado, o que nem sempre é uma tarefa fácil. Cabe ressaltar que a precisão de ambas as operações depende em grande medida da habilidade do operador, o qual deverá estar capacitado para realização destas tarefas, caso contrário, outras fontes de erros podem ser associadas e resultar em uma contribuição significativa para a incerteza da medição.

Por outro lado, os parâmetros que definem a geometria do cordão são determinados a partir de corpos de prova, os quais devem ser cortados e preparados de forma cuidadosa a fim de garantir a sua qualidade geométrica. Geralmente, serras de disco são usadas para efetuar um corte transversal ao cordão de solda. Apesar dos avanços tecnológicos, a obtenção de superfícies perfeitas geometricamente constitui, ainda hoje, um grande desafio. Até as mais modernas máquinas-ferramenta apresentam desvios geométricos indesejáveis que podem comprometer as dimensões e a geometria das peças usinadas, criando a necessidade de quantificar e indicar as tolerâncias geométricas nas peças e desenvolver meios para verificá-las. Os desvios de forma são definidos como o grau de variação das superfícies reais com relação às geometrias ideais que as definem [12].

Uma análise dos corpos de prova revela que os desvios de perpendicularidade e de planeza são aqueles que podem alterar os valores dos parâmetros geométricos do cordão de forma mais acentuada. A perpendicularidade (figura 1A) é uma tolerância geométrica de orientação e é aplicada a dois ou mais elementos, tendo sempre um deles como referência para o posicionamento perpendicular dos demais [12]. Assim, o desvio de perpendicularidade dos corpos de prova pode ser definido como o ângulo formado entre o plano obtido durante o corte e um plano de referência formado pelas chapas soldadas. Da geometria analítica se conhece que somente um corte efetuado a $90^{\circ}$ permitirá revelar o valor verdadeiro da penetração, por exemplo, e que qualquer ângulo diferente gerará valores sempre maiores. Ainda, pode ser avaliado o desvio de perpendicularidade entre o plano de medição e a superfície lateral de uma das chapas soldadas (figura 1B).

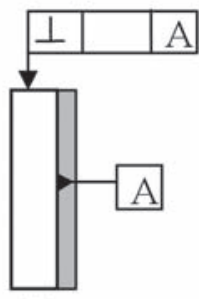

(A)

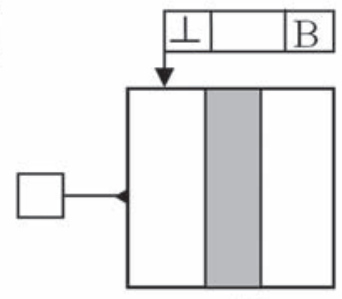

(B)

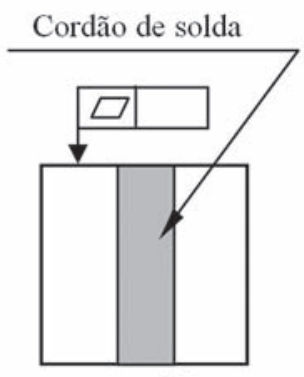

(C)
Figura 1. Tolerâncias de planeza e de perpendicularidade.

Na preparação dos corpos de prova, tanto o lixamento quanto o polimento são realizados de forma manual, o que pode provocar ondulações na superfície se o esforço exercido sobre ela não for homogêneo. Estas ondulações podem ser caracterizadas pelo desvio planeza. Segundo a NBR 6409 (1997) [12] o campo de tolerância para a planeza é limitado por dois planos paralelos distantes de $t$, entre os quais deve estar situada a superfície considerada, figura $1 \mathrm{C}$.

Este trabalho tem como objetivo apresentar uma metodologia para estimar a incerteza associada à medição dos parâmetros que definem a geometria de cordões de solda, especificamente a área de reforço, conforme recomendado pelo ISO GUM [13]. Adicionalmente, será avaliada a qualidade dos corpos de prova utilizados durante as medições. Espera-se com isto contribuir para a adequação do processo de medição à exigências da norma NBR ISO/IEC 17025 [14].

\section{Materiais e Métodos}

Com o intuito de aplicar o procedimento de cálculo da incerteza à medição dos parâmetros geométricos de um depósito de soldagem, foram utilizadas duas placas de aço 35ХГС [15] com dimensões de 150 × 50 × $6 \mathrm{~mm}$. Estas placas foram limpas para eliminar óxidos e outras impurezas. A fixação durante a solda foi feita com ajuda de um suporte mecânico, para evitar deformações devido ao ciclo térmico.

Como material de enchimento, foi utilizado um eletrodo revestido E 11018-M, segundo a classificação AWS A 5.52004, com diâmetro e comprimento de $3,25 \mathrm{~mm}$ e $350,00 \mathrm{~mm}$, respectivamente. A partir das recomendações do fabricante do eletrodo e considerando que os depósitos seriam realizados em posição plana, foi utilizado um nível de corrente de soldagem médio de 130 A. Foi adotado, como fonte de potência, um retificador trifásico marca Mansfeld, modelo G $500 \mathrm{VC} / \mathrm{S}$, com tensão em vazio de $74 \mathrm{~V}$.

A velocidade média de $15 \mathrm{~cm} / \mathrm{min}$ foi estimada em função do tempo do arco e do comprimento do cordão. O tempo de arco foi medido com um cronômetro de resolução de $0,1 \mathrm{~s}$, enquanto que para a medição do comprimento foi usado um Paquímetro de resolução $0,02 \mathrm{~mm}$.

O procedimento de soldagem foi conduzido no Laboratório de Tecnologia de Soldagem do Centro de Pesquisas de Soldagem da Universidade Central "Marta Abreu" de Las Villas, por um soldador qualificado e com experiência no processo SMAW. 
Após a realização dos depósitos, as placas foram limpas para remover escórias e respingos. Ambos os cordões foram cortados transversalmente, em sete seções cada, usando uma serra circular com disco abrasivo. Cabe ressaltar que foram desprezados $25 \mathrm{~mm}$ nos extremos de cada cordão, para evitar as regiões de instabilidade do arco e rechupes no início e no fim do cordão, respectivamente.

Posteriormente se procedeu à preparação dos corpos de prova visando à determinação dos parâmetros que definem a geometria do cordão. Para tanto, foi aplicado o procedimento CIS-MET-030 [16] desenvolvido pelo Laboratório de Diagnóstico e Prognóstico de Falhas. Desta forma, os corpos de prova foram montados em acrílico para facilitar a manipulação e evitar deformações da superfície. Em seguida, foram atacados com Nital $2 \%$, segundo o procedimento CIS-MET-027 [17].

\subsection{Medição dos parâmetros do cordão de solda}

Para medição dos parâmetros geométricos do cordão foi utilizado um sistema de captação de imagens NOVEL modelo NIM-100. Inicialmente, o sistema foi ajustado até obter uma iluminação uniforme e melhorar o contraste. Em seguida, o sistema óptico foi calibrado utilizando uma régua de aço, com resolução de $0,5 \mathrm{~mm}$, conforme proposto por CATTANI et al. (2008) [18]. Esta calibração consistiu das seguintes etapas: captação da imagem da régua; projeção, na tela do monitor, da imagem da régua para ser utilizada como referência; traçagem de uma linha com comprimento igual à menor divisão da régua $(0,5 \mathrm{~mm})$; determinação do número de pixels ocupado pela linha que separa dois traços consecutivos da escala da régua. Esta operação se repetiu para uma linha equivalente à metade, a um quarto, a um oitavo e a um dezesseis avos do comprimento da menor divisão. Em cada caso, o número de pixels ocupado por estas linhas foi determinado e após cinco medições, bem como, a dispersão dos valores. Ao concluir esta etapa, foram obtidas as imagens dos corpos de prova para seu posterior processamento por meio do programa computacional SigmaScan Pro versão 5.0. Este programa permite realizar as medições com base na relação entre o número de pixels e a dimensão real, tomando como referência a imagem padrão.

\subsection{Medição dos Desvios Geométricos}

Os desvios de planeza e de perpendicularidade dos corpos de prova foram medidos com uma Máquina de Medir por Coordenadas (MMC) do tipo Ponte Móvel. Esta máquina do fabricante MITUTOYO possui resolução de $1 \mu \mathrm{m}$ e volume de trabalho de $400 \times 400 \times 300 \mathrm{~mm}$ para os eixos X, Y e Z, respectivamente. Durante a medição foi utilizado um único apalpador, com esfera de rubi de diâmetro igual a $2 \mathrm{~mm}$ (figura 2).

Ambos os desvios geométricos foram medidos três vezes em cada corpo de prova. Todos os planos de medição foram definidos a partir de 15 pontos, determinados com a MMC. Simultaneamente, a temperatura ambiente foi monitorada com um termo-higrômetro digital de resolução $0,1^{\circ} \mathrm{C}$ e faixa nominal de -20 a $60^{\circ} \mathrm{C}$. As medições foram efetuadas no Laboratório de Metrologia Dimensional de Universidade Federal de Uberlândia, na temperatura de $20 \pm 1{ }^{\circ} \mathrm{C}$.

\subsection{Estimativa da Incerteza de Medição}

A incerteza associada à medição dos desvios geométricos e da área de reforço foi estimada, uma vez que resultados de medições expressos apenas como a média aritmética carecem de significado por não fornecer a informação completa sobre
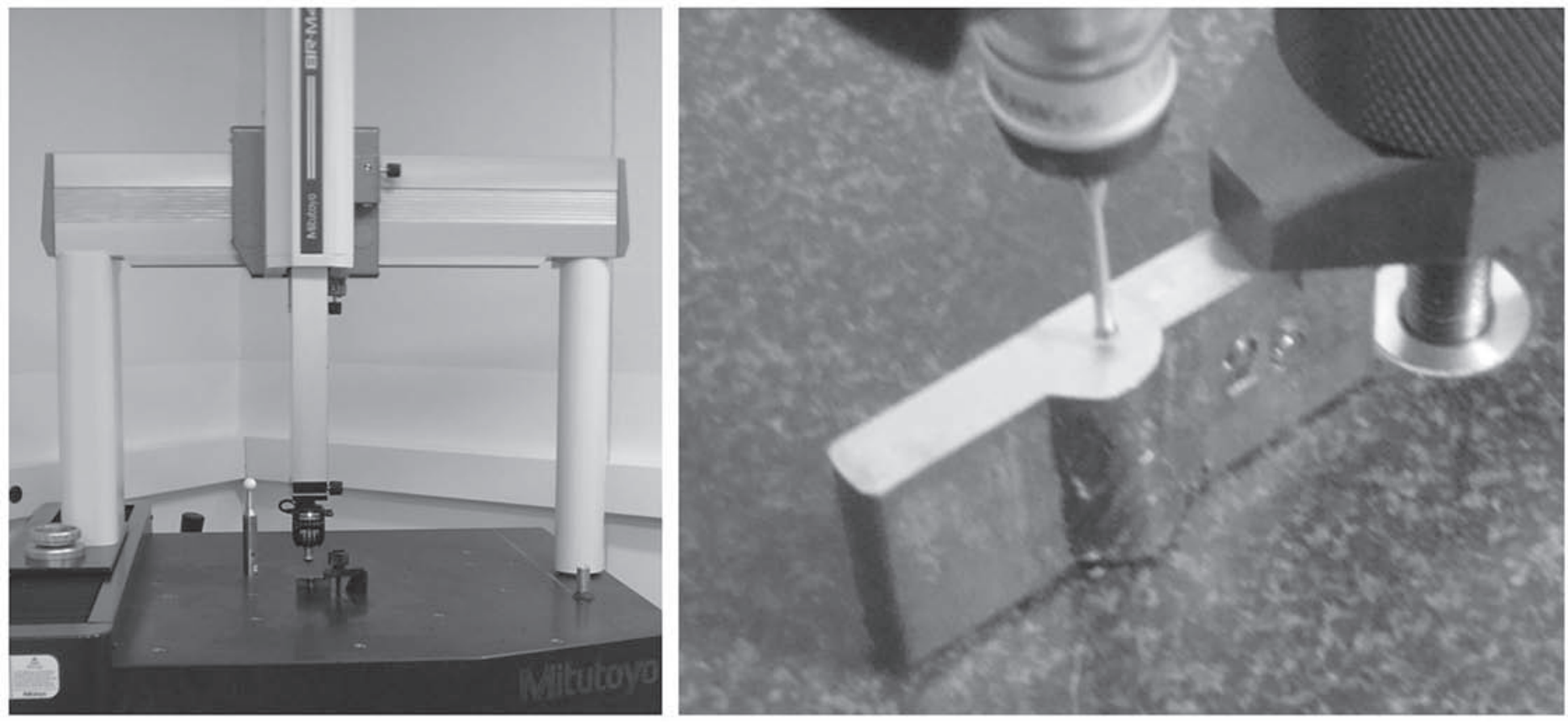

Figura 2. Montagem experimental para medição dos desvios de perpendicularidade e de planeza com uma MMC. 

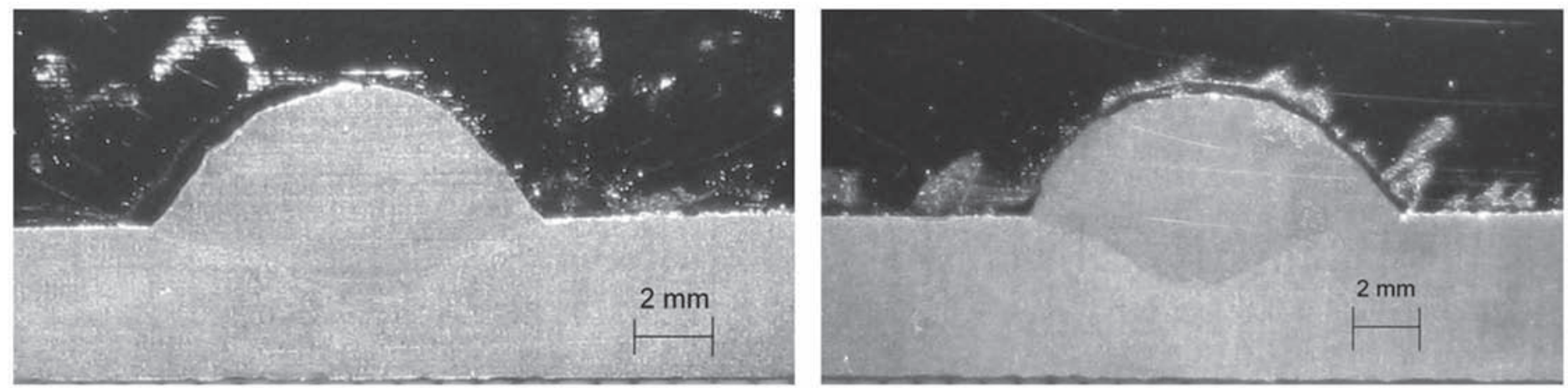

Figura 3. Imagens dos corpos de prova C6 (à esquerda) e C12 (à direita).

a medição. Mesmo que o desvio padrão seja declarado junto com a média, a maior parte das variáveis de influência não seria considerada. Dentre estas, pode-se mencionar a incerteza relacionada à calibração do sistema de medição, aos desvios geométricos dos corpos de prova e ao afastamento da temperatura em relação a $20^{\circ} \mathrm{C}$.

\section{Resultados e Discussão}

A seguir são apresentados os resultados encontrados e suas respectivas discussões.

\subsection{Parâmetros do cordão de solda}

A figura 3 mostra as imagens captadas dos corpos de prova 6 e 12. Observe que existe uma zona de fusão incompleta nos dois corpos de prova, na qual ocorrem processos difusivos entre o metal fundido e o metal base, dificultando a definição dos limites entre estes. Devido a esta indefinição de contorno, podem ser observadas diferenças significativas entre os valores da penetração e das áreas medidas por operadores diferentes.

A seguir foram determinadas as dimensões lineares (penetração, largura e reforço) e as áreas de reforço e de penetração. Cada um dos parâmetros foi medido três vezes para cada corpo de prova ( $\mathrm{C} 1$ a $\mathrm{C} 12)$ e as médias aritméticas das leituras foram calculadas (tabela 1).

Tabela 1. Valores dos parâmetros que definem a geometria do cordão.

\begin{tabular}{|c|c|c|c|c|c|}
\hline & $\begin{array}{c}\text { Largura } \\
(\mathrm{mm})\end{array}$ & $\begin{array}{c}\text { Reforço } \\
(\mathrm{mm})\end{array}$ & $\begin{array}{c}\text { Penetração } \\
(\mathrm{mm})\end{array}$ & $\begin{array}{c}\text { Área } \\
\text { Reforço } \\
\left(\mathrm{mm}^{2}\right)\end{array}$ & $\begin{array}{c}\text { Área } \\
\text { Penetração } \\
\left(\mathrm{mm}^{2}\right)\end{array}$ \\
\hline C1 & 10,02 & 2,89 & 1,45 & 27,28 & 11,56 \\
\hline C2 & 9,30 & 1,74 & 1,53 & 15,87 & 7,76 \\
\hline C3 & 10,67 & 2,85 & 1,74 & 18,56 & 10,24 \\
\hline C4 & 10,15 & 3,69 & 1,42 & 24,58 & 9,38 \\
\hline C5 & 9,80 & 3,06 & 2,23 & 20,46 & 10,54 \\
\hline C6 & 9,91 & 3,34 & 1,41 & 21,27 & 9,17 \\
\hline C7 & 8,34 & 3,10 & 1,17 & 17,26 & 11,48 \\
\hline C8 & 10,59 & 3,06 & 2,04 & 20,69 & 7,76 \\
\hline C9 & 9,96 & 2,91 & 1,60 & 19,23 & 8,70 \\
\hline C10 & 9,58 & 3,77 & 1,47 & 20,13 & 8,29 \\
\hline C11 & 9,85 & 2,97 & 1,67 & 19,67 & 7,63 \\
\hline C12 & 10,65 & 3,46 & 2,05 & 26,13 & 10,45 \\
\hline
\end{tabular}

Apesar de haver diferenças entre as dimensões encontradas para os doze corpos de prova ( $\mathrm{C} 1$ a $\mathrm{C} 12)$, os valores observados são considerados adequados, sobretudo se comparadas às médias aritméticas de cada parâmetro medido. Com relação à variabilidade das leituras, pode-se dizer que foi maior para as áreas determinadas. Isto era esperado e pode ser justificado pela dificuldade em definir os limites das mesmas durante a medição.

\subsection{Desvios Geométricos}

Na figura 4, são apresentados os valores médios encontrados para o desvio de planeza dos corpos de prova. Nesta figura, pode ser observado que estes desvios apresentam uma variação entre 4 e $11 \mu \mathrm{m}$, sendo que o corpo de prova $\mathrm{C} 1$ apresentou um valor relativamente maior que os demais. Espera-se que estes valores pouco influenciem os parâmetros geométricos do cordão de solda.

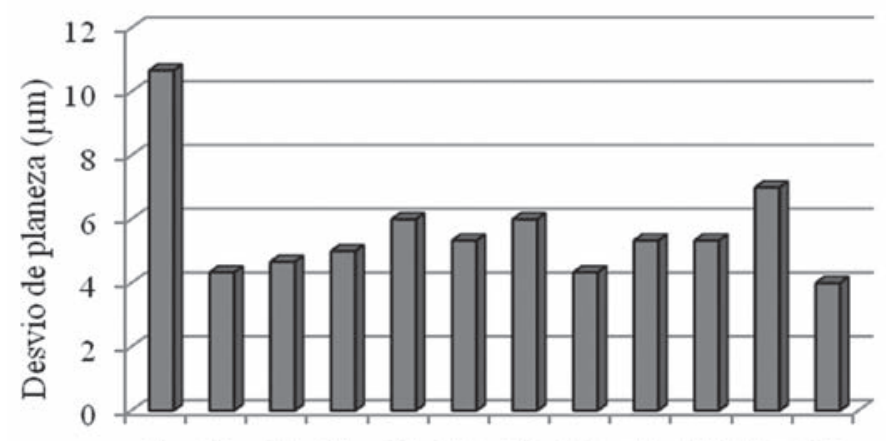

$\begin{array}{llllllllllllllll}\mathrm{C} 1 & \mathrm{C} 2 & \mathrm{C} 3 & \mathrm{C} 4 & \mathrm{C} 5 & \mathrm{C} 6 & \mathrm{C} 7 & \mathrm{C} 8 & \mathrm{C} 9 & \mathrm{C} 10 & \mathrm{C} 11 & \mathrm{C} 12\end{array}$

Figura 4. Desvios de planeza dos corpos de prova $\mathrm{C} 1$ a $\mathrm{C} 12$.

Na figura 5, são mostrados os valores do desvio de perpendicularidade no sentido da espessura e da largura da chapa, para os seis corpos de prova retirados do cordão de solda 1. Nesta figura, pode ser observado que os valores da perpendicularidade variam de forma significativa de um corpo de prova para o outro, assumindo valores entre $15^{\prime}$ e $1^{\circ} 41^{\prime}$. A perpendicularidade na direção da largura das chapas apresentou valores relativamente maiores, quando comparado com a perpendicularidade na direção da espessura da chapa. Assim sendo, é esperado que a contribuição do referido desvio no valor da largura do cordão de solda e na incerteza de medição seja mais significativa, pois este parâmetro apresenta os maiores valores dentre os parâmetros lineares. 
Para os corpos de prova do segundo cordão (C7-C12), os valores de perpendicularidade apresentaram uma variabilidade maior, assumindo valores entre $1^{\circ} 9^{\prime}$ e $2^{\circ} 41^{\prime}$.

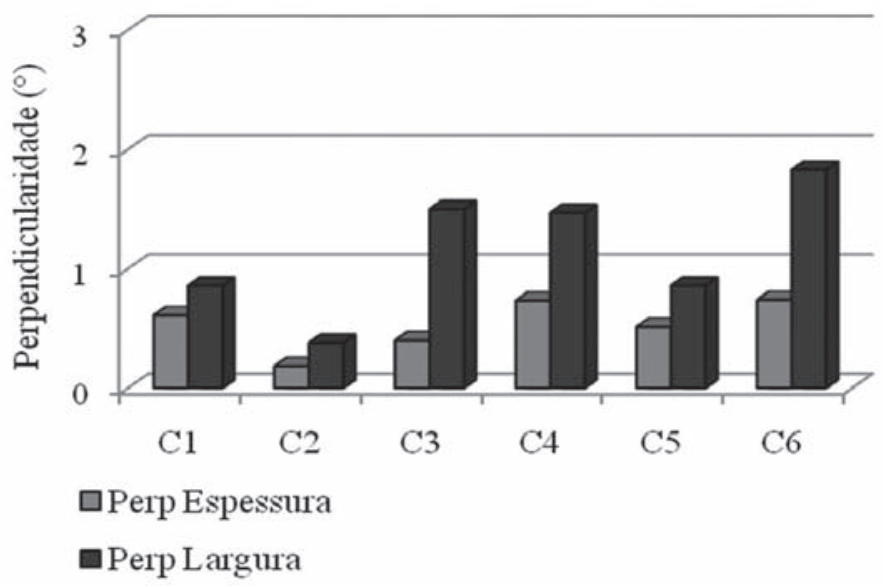

Figura 5. Desvio de perpendicularidade do cordão 1 (corpos de prova $\mathrm{C} 1-\mathrm{C} 6)$.

\subsection{Cálculo da incerteza dos desvios de planeza e de perpendicularidade}

Para estimativa da incerteza da medição de perpendicularidade, foram consideradas as seguintes variáveis: indicação ou leitura, resolução, erro de apalpamento e a incerteza associada à calibração da MMC. Os valores referentes às duas últimas variáveis foram extraídos do certificado de calibração da MMC. Por sua vez, para estimar a incerteza associada ao desvio de planeza, foi considerado inclusive o desvio de planeza do desempeno da MMC. O cálculo da incerteza não contemplou os efeitos térmicos, que foram desconsiderados porque as medições foram efetuadas a $20 \pm 1^{\circ} \mathrm{C}$ e os valores dos desvios associados foram muito pequenos.

Os valores de incerteza expandida associados à medição do desvio de planeza variaram entre 5,3 e 6,8 $\mu \mathrm{m}$ para os diferentes corpos de prova. Por sua vez, os valores de incerteza para a perpendicularidade se encontraram entre 8 e $194 \mu \mathrm{m}$. Para ambos, a probabilidade de abrangência foi adotada como 95,45 $\%$.

\subsection{Estimativa da Incerteza Associada à Medição dos Parâmetros do Cordão de Solda}

Uma análise do cordão revelou que o conjunto de variáveis que contribui para a incerteza de medição difere para cada parâmetro geométrico estudado. Considerando que os corpos de prova foram posicionados na mesa da MMC e que o sistema de coordenadas foi transferido para a peça (origem no ponto 0), os parâmetros do cordão de solda podem ser indicados como mostra a figura 6 . Nesta figura, o valor b representa a largura do cordão, hr corresponde à altura, hp é a penetração, Ar é a área de reforço e Ap é a área de penetração.

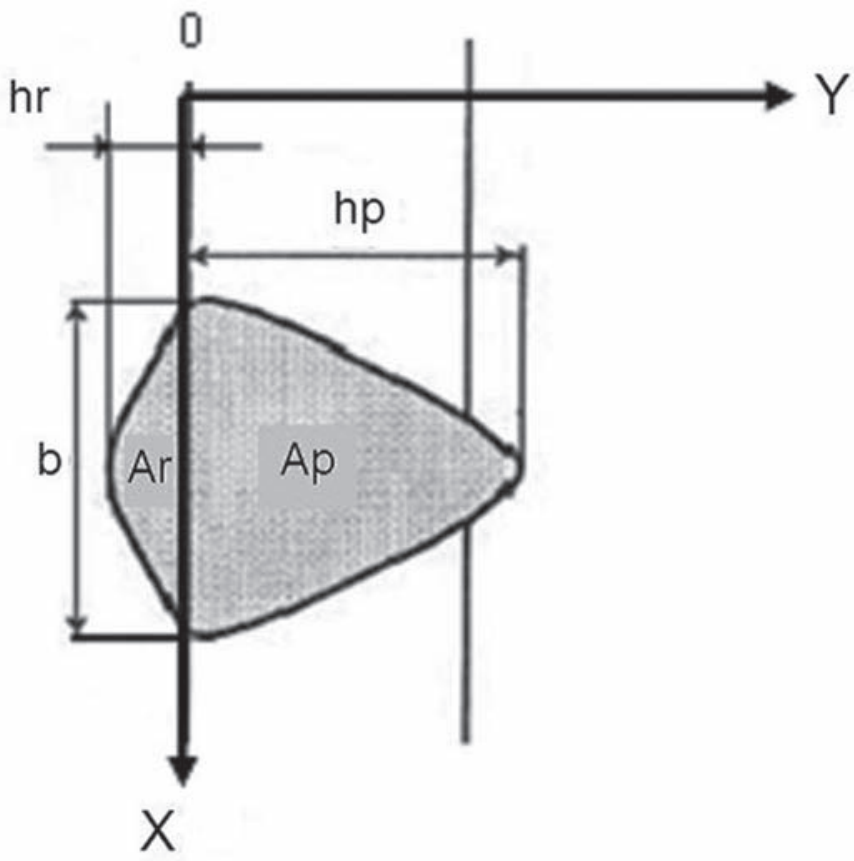

Figura 6. Parâmetros do cordão.

A relação entre as variáveis de influência e os parâmetros do cordão está apresentada na tabela 2. Nesta tabela, os símbolos correspondem às seguintes variáveis: $\mathrm{L}$ equivale às leituras do parâmetro em questão; $\mathrm{Pl}_{\mathrm{CP}}, \mathrm{P} 1_{\mathrm{CP}}$ e $\mathrm{P} 2_{\mathrm{CP}}$ representam respectivamente o desvio de planeza $(\mathrm{P} 1)$ e de perpendicularidade (P2) dos corpos de prova nas duas direções consideradas; $\mathrm{R}_{\mathrm{SM}}$ representa a resolução do sistema de medição, $\mathrm{IC}_{\mathrm{SM}}$ é a incerteza associada à calibração do sistema de medição, $\Delta \mathrm{T}$ é o afastamento da temperatura ambiente com relação a $20^{\circ} \mathrm{C}$ e $\delta \mathrm{T}$ é a variação da temperatura ambiente durante a medição.

Tabela 2. Variáveis de influência na medição de todos oe parâmetros do cordão de solda.

\begin{tabular}{|c|c|c|c|c|c|c|c|c|}
\hline Variáveis & \multirow{2}{*}{$\mathrm{L}$} & \multirow{2}{*}{$\mathrm{Pl}_{\mathrm{CP}}$} & \multirow{2}{*}{$\mathrm{PT}_{\mathrm{CP}}$} & \multirow{2}{*}{$\mathrm{P}_{\mathrm{CP}}$} & \multirow{2}{*}{$\mathrm{R}_{\mathrm{SM}}$} & \multirow{2}{*}{$\mathrm{IC}_{\mathrm{SM}}$} & \multirow{2}{*}{$\Delta \mathrm{T}$} & \multirow{2}{*}{$\delta \mathrm{T}$} \\
\hline Parâmetros & & & & & & & & \\
\hline b (largura) & $\mathrm{X}$ & & & $\mathrm{X}$ & $\mathrm{X}$ & $\mathrm{X}$ & $\mathrm{X}$ & $\mathrm{X}$ \\
\hline hr (altura) & $\mathrm{X}$ & & $X$ & & $\mathrm{X}$ & $\mathrm{X}$ & $\mathrm{X}$ & $\mathrm{X}$ \\
\hline $\begin{array}{l}\text { hp } \\
\text { (penetração) }\end{array}$ & $X$ & & $\mathrm{X}$ & & X & $\mathrm{X}$ & $\mathrm{X}$ & $\mathrm{X}$ \\
\hline $\begin{array}{l}\text { Ar (área de } \\
\text { reforço) }\end{array}$ & $\mathrm{X}$ & $\mathrm{X}$ & $\mathrm{X}$ & $\mathrm{X}$ & X & $\mathrm{X}$ & $\mathrm{X}$ & $\mathrm{X}$ \\
\hline $\begin{array}{l}\text { Ap área de } \\
\text { penetração) }\end{array}$ & $\mathrm{X}$ & $\mathrm{X}$ & $\mathrm{X}$ & $\mathrm{X}$ & $\mathrm{X}$ & $\mathrm{X}$ & $\mathrm{X}$ & $\mathrm{X}$ \\
\hline
\end{tabular}

Neste trabalho foram medidos cinco parâmetros para caracterizar a geometria do cordão, entretanto, será apresentada a metodologia completa para estimar a incerteza associada à medição da área de penetração. Este parâmetro foi escolhido porque o processo de medição apresenta as maiores inexatidões devido à não existência de uma zona perfeitamente definida e à correlação entre as variáveis envolvidas no cálculo. 
A equação (1) é utilizada para obtenção da área de reforço. As variáveis $\mathrm{a}_{\mathrm{Ar}}$ e $\mathrm{b}_{\mathrm{Ar}}$ representam a largura e o comprimento do quadrado de $1 \mathrm{~mm}^{2}$ de área tomado como referência. Essas variáveis estão diretamente correlacionadas, assim sendo, a incerteza padrão combinada associada à medição da área de reforço pode ser calculada pela equação (2). O coeficiente de correlação, nesse caso, assume o valor +1 .

$$
\begin{aligned}
& A_{\gamma}=a_{A \gamma} \cdot b_{A \gamma} \\
& u^{2}\left(A_{\gamma}\right)=\left(\frac{\partial A_{\gamma}}{\partial a_{A \gamma}}\right)^{2} \cdot u^{2}\left(a_{A \gamma}\right)+\left(\frac{\partial A_{\gamma}}{\partial b_{A r}}\right)^{2} \cdot u^{2}\left(b_{A r}\right)
\end{aligned}
$$

A incerteza associada à $a_{A r}$ e à $b_{A r}$ pode ser calculada utilizando as equações (3) e (4), respectivamente.

$$
\begin{aligned}
& a_{A r}=L a_{A r}+P_{C P}+R_{S M}+I C_{S M}+\Delta T_{20}+\delta T \\
& b_{A r}=L b_{A r}+P T_{C P}+R_{S M}+I C_{S M}+\Delta T_{20}+\delta T
\end{aligned}
$$

A lei de propagação de incertezas pode ser aplicada em (3 e 4), e assim as incertezas padrão combinadas de $\mathrm{a}_{\mathrm{Ar}}$ e $\mathrm{b}_{\mathrm{Ar}}$ podem ser determinadas pelas expressões (5) e (6), respectivamente.

$$
\begin{aligned}
& u_{c}^{2}\left(a_{A v}\right)=\left(\frac{\partial a_{A v}}{\partial L_{A v}}\right)^{2} u^{2}\left(L_{A v}\right)+\left(\frac{\partial a_{A v}}{\partial P T_{C P}}\right)^{2} u^{2}\left(P T_{C P}\right)+ \\
& \left(\frac{\partial a_{A v}}{\partial R_{S M}}\right)^{2} u^{2}\left(R_{S M}\right)+\left(\frac{\partial a_{A v}}{\partial I C_{S M}}\right)^{2} u^{2}\left(I C_{S M}\right)+ \\
& +\left(\frac{\partial a_{A v}}{\partial \Delta T_{20}}\right)^{2} u^{2}\left(\Delta T_{20}\right)+\left(\frac{\partial a_{A v}}{\partial \delta T}\right)^{2} u^{2}(\delta T) \\
& u_{c}^{2}\left(b_{A v}\right)=\left(\frac{\partial b_{A v}}{\partial L_{A v}}\right)^{2} u^{2}\left(L_{A v}\right)+\left(\frac{\partial b_{A v}}{\partial P T_{C P}}\right)^{2} u^{2}\left(P T_{C P}\right)+ \\
& \left(\frac{\partial b_{A v}}{\partial R_{S M}}\right)^{2} u^{2}\left(R_{S M}\right)+\left(\frac{\partial b_{A v}}{\partial I C_{S M}}\right)^{2} u^{2}\left(I C_{S M}\right)+ \\
& +\left(\frac{\partial b_{A v}}{\partial \Delta T_{20}}\right)^{2} u^{2}\left(\Delta T_{20}\right)+\left(\frac{\partial b_{A v}}{\partial \delta T}\right)^{2} u^{2}(\delta T)
\end{aligned}
$$

Para calcular estas incertezas padrão combinadas, a incerteza padrão de cada variável individual deve ser determinada conforme apresentado nas equações (7) a (11).

\section{Incerteza padrão associada às Leituras}

Devido ao caráter aleatório do processo de medição sempre haverá uma incerteza padrão associada à variabilidade das leituras. Esta incerteza pode ser estimada pela equação (7) através de uma avaliação do Tipo A. A distribuição de probabilidades associada é a distribuição normal, com n-1 graus de liberdade.

$$
u\left(L a_{A \gamma}\right)=\sqrt{\frac{s^{2}(\text { Leitura })}{n}}
$$

\section{Incerteza padrão associada à Resolução do Sistema de Medição}

A incerteza padrão associada à resolução do sistema de medição é do Tipo B e pode ser estimada segundo a equação (8), admitindo uma distribuição de probabilidades retangular, com $\infty$ graus de liberdade. A resolução do sistema de medição é de $0,03 \mathrm{~mm}$.

$$
u\left(R_{S M}\right)=\frac{R_{S M}}{\sqrt{3}}
$$

\section{Incerteza padrão associada à calibração do Sistema de medição}

Esta incerteza foi estimada a partir dos dados da calibração do sistema de medição. $O$ valor encontrado foi de $0,496 \mathrm{~mm}$ para um intervalo de abrangência de $95,5 \%$ e $\mathrm{k}=2,2$. Assim sendo, para padronizar essa incerteza basta dividir pelo valor $\mathrm{k}$ (equação 9).

$$
u\left(C_{S M}\right)=\frac{U p(\text { da calibração })}{k}
$$

Incerteza padrão dos desvios de planeza e de perpendicularidade

A incerteza padrão associada à contribuição dos desvios de planeza e de perpendicularidade em cada um dos parâmetros geométricos do cordão é do Tipo $\mathrm{B}$ e pode ser estimada segundo as equações (10) e (11), admitindo uma distribuição de probabilidade normal com n-1 graus de liberdade.

$$
\begin{aligned}
& u\left(D_{P l}\right)=\frac{D_{P L}}{\sqrt{n}} \\
& u\left(D_{P}\right)=\frac{C\left(D_{P}\right)}{\sqrt{n}}
\end{aligned}
$$

Cabe ressaltar que na equação (10) foi considerado o valor total do desvio de planeza para efetuar o cálculo. Entretanto, na equação (11) foi considerada a parcela de contribuição do desvio de penpendicularidade. Assim, a equação (3) pode ser reescrita, obtendo a equação (12), onde $\alpha$ representa o ângulo equivalente ao desvio de perpendicularidade entre as superfícies dos corpos de prova.

$$
a_{A r}=L a_{A r}+L(1-\cos \alpha)+R_{S M}+I C_{S M}+\Delta T_{20}+\delta T
$$

\section{Estimativa da incerteza padrão combinada $\left(u_{c}\right)$}

Uma vez estimadas as incertezas-padrão das variáveis presentes nas equações (3) e (4), a incerteza padrão combinada das variáveis $\mathrm{a}_{\mathrm{Ar}}$ e $\mathrm{b}_{\mathrm{Ar}}$ deve ser calculada utilizando as equações (5) e (6). Em seguida, a incerteza padrão combinada associada à área de reforço pode ser obtida substituindo os valores na equação (12).

\section{Estimativa da incerteza expandida (Up)}

A incerteza expandida associada à área de reforço deve ser calculada pela equação (13).

$$
U p(A r)=k \cdot u_{C}(A r)
$$


Para determinar o valor do fator de abrangência, deve ser utilizada a tabela da distribuição t de Student. O número de graus de liberdade efetivo $\left(v_{\text {efetivo }}\right)$ deve ser determinado pela equação (14), onde $u_{\mathrm{C}}^{4}(\mathrm{y})$ corresponde à incerteza padrão combinada da área de reforço e $u_{i}\left(x_{i}\right)$ representa a incerteza padrão das variáveis de entrada.

$$
v_{\text {efetivo }}=\frac{u_{c}^{4}(y)}{\sum\left(\frac{u_{i}^{4}\left(x_{i}\right)}{v_{i}}\right)}
$$

Todas as informações referentes ao cálculo da incerteza de $\mathrm{a}_{\mathrm{Ar}}$ e $\mathrm{b}_{\mathrm{Ar}}$ são apresentadas de forma resumida nas tabelas 3 e 4 . Três algarismos foram acrescentados para minimizar a propagação de erros.

Observe, nas tabelas 3 e 4, que a variável que mais contribuiu com a incerteza final de $a_{\mathrm{Ar}}$ e $\mathrm{b}_{\mathrm{Ar}}$ foi a incerteza associada à calibração do sistema de medição. Este resultado pode ser justificado porque o padrão utilizado para calibrar o sistema óptico foi uma régua de aço de resolução de $0,5 \mathrm{~mm}$. A contribuição desta variável pode ser reduzida utilizando-se um padrão com resolução maior, por exemplo, de $0,01 \mathrm{~mm}$.
Para o corpo de prova 1 , em particular, a contribuição do desvio de perpendicularidade na incerteza de $\mathrm{a}_{\mathrm{Ar}}$ não foi significativa, entretanto, para $b_{A r}$ a parcela de contribuição deste desvio ficou em segundo lugar em importância. Desta forma, se uma régua com resolução melhor que $0,5 \mathrm{~mm}$ for utilizada para calibrar o sistema óptico, a influência do desvio de perpendicularidade pode se tornar importante.

\section{Cálculo da incerteza padrão combinada e expandida de Ar}

Uma vez conhecidas as incertezas padrão de $a_{\mathrm{Ar}}$ e $b_{\mathrm{Ar}} \mathrm{p}$, deve ser feito o cálculo da incertezas associadas à medição da área de reforço. Substituindo os valores na equação (2), a incerteza padrão combinada resultante foi igual a $0,35532 \mathrm{~mm}^{2}$, enquanto que a incerteza expandida foi igual a $1,01975 \mathrm{~mm}^{2}$ para $\mathrm{k}=2,87$ e $95,45 \%$ de abrangência.

De forma similar, a incerteza deve ser estimada para a totalidade dos corpos de prova. Os resultados obtidos estão apresentados na tabela 5, para os corpos de prova dos cordões 1 e 2. Para o corpo de prova 1 , a área de reforço foi equivalente a $27,28 \pm 0,66 \mathrm{~mm}^{2}$, com incerteza expandida obtida adotando uma probabilidade de abrangência de 95,45 \% e k $=2,28$. Todos os resultados das medições devem ser apresentados desta maneira, para que estejam de acordo com as normas em vigor.

Tabela 3. Incerteza associada à $\mathrm{a}_{\mathrm{Ar}}$ para o corpo de prova 1 .

\begin{tabular}{lccccc}
\hline $\begin{array}{l}\text { Grandeza } \\
\mathrm{X}_{\mathrm{i}}\end{array}$ & Estimativa $\mathrm{x}_{\mathrm{i}}$ & $\begin{array}{c}\text { Distribuição } \\
\text { de probabilidade }\end{array}$ & $\begin{array}{c}\text { Coeficiente de } \\
\text { sensibilidade }\end{array}$ & $\begin{array}{c}\text { Graus de } \\
\text { liberdade }\end{array}$ & $\begin{array}{c}\text { Incerteza padrão } \\
(\mathrm{mm})\end{array}$ \\
\hline $\mathrm{La}_{\mathrm{Ar}}$ & $10,02333 \mathrm{~mm}$ & Normal & 1 & 2 & 0,00667 \\
$\mathrm{R}_{\mathrm{SM}}$ & $0,03000 \mathrm{~mm}$ & Retangular & 1 & $\square$ & 0,01732 \\
$\mathrm{IC}_{\mathrm{SM}}$ & $0,49500 \mathrm{~mm}$ & Normal & 1 & 4 & 0,22500 \\
$\alpha$ & $0,01065 \mathrm{rad}$ & Normal & $19,04616 \mathrm{~mm}$ & 2 & 0,00004 \\
$\mathrm{~L}$ & $10,02276 \mathrm{~mm}$ & Normal & $0,10671 \mathrm{rad}$ & 2 & 0,00667 \\
\hline Incerteza padrão combinada $\left(\mathrm{u}_{\mathrm{c}}\right) \mathrm{em} \mathrm{mm}$ & & & 0,25902 \\
\hline \multicolumn{2}{l}{ Grau de liberdade efetivo $\left(v_{\text {ef }}\right)$} \\
\multicolumn{2}{l}{ Fator de abrangência $(95,45 \%)$} \\
Incerteza expandida $(\mathrm{Up}) \mathrm{em} \mathrm{mm}$
\end{tabular}

Tabela 4. Incerteza associada $a b_{A r}$ para o corpo de prova 1 .

\begin{tabular}{|c|c|c|c|c|c|}
\hline $\begin{array}{c}\text { Grandeza } \\
X_{i}\end{array}$ & Estimativa $x_{i}$ & $\begin{array}{c}\text { Distribuição } \\
\text { de probabilidade }\end{array}$ & $\begin{array}{c}\text { Coeficiente de } \\
\text { sensibilidade }\end{array}$ & $\begin{array}{l}\text { Graus de } \\
\text { liberdade }\end{array}$ & $\begin{array}{l}\text { Incerteza padrão } \\
\qquad(\mathrm{mm})\end{array}$ \\
\hline $\mathrm{Lb}_{\mathrm{Ar}}$ & $2,89333 \mathrm{~mm}$ & Normal & 1 & 2 & 0,01856 \\
\hline $\mathrm{R}_{\mathrm{cu}}$ & $0,03000 \mathrm{~mm}$ & Retangular & 1 & $\square$ & 0,01732 \\
\hline $\begin{array}{l}\mathrm{IC}_{\mathrm{SM}} \\
\mathrm{IC}_{\mathrm{SM}}\end{array}$ & $0,49500 \mathrm{~mm}$ & Normal & 1 & 4 & 0,22500 \\
\hline$\alpha^{\mathrm{SM}}$ & $0,01501 \mathrm{rad}$ & Normal & $4,78589 \mathrm{~mm}$ & 2 & 0,00000 \\
\hline $\mathrm{L}$ & $2,89301 \mathrm{~mm}$ & Normal & $0,06160 \mathrm{rad}$ & & 0,08882 \\
\hline \multicolumn{5}{|c|}{ Incerteza padrão combinada $\left(u_{c}\right)$ em mm } & 0,24323 \\
\hline \multicolumn{5}{|c|}{ Grau de liberdade efetivo $\left(v_{e f}\right)$} & 5,46 \\
\hline \multicolumn{5}{|c|}{ Fator de abrangência $(95,45 \%)$} & $\mathrm{k}=2,58$ \\
\hline \multicolumn{5}{|c|}{ Incerteza expandida (Up) em mm } & 0,62752 \\
\hline
\end{tabular}


Tabela 5. Resultados da medição das áreas de reforço (Ar).

\begin{tabular}{lcccccc}
\hline \multicolumn{7}{c}{ Corpos de prova do cordão 1 } \\
\hline & $\mathrm{C} 1$ & $\mathrm{C} 2$ & $\mathrm{C} 3$ & $\mathrm{C} 4$ & $\mathrm{C} 5$ & $\mathrm{C} 6$ \\
\hline $\bar{x}\left(\mathrm{~mm}^{2)}\right.$ & 27,28 & 15,87 & 18,56 & 24,58 & 20,46 & 21,27 \\
$\mathrm{u}_{\mathrm{c}}\left(\mathrm{mm}^{2}\right)$ & 0,36 & 0,38 & 0,38 & 0,52 & 0,52 & 0,37 \\
$\mathrm{k}$ & 2,28 & 2,09 & 2,13 & 2,00 & 2,00 & 2,16 \\
$\mathrm{Up}\left(\mathrm{mm}^{2)}\right.$ & 1,02 & 1,15 & 1,09 & 1,71 & 1,72 & 1,06 \\
\hline \multicolumn{7}{c}{ Corpos de prova do cordão 2} \\
\hline $\bar{x}$ & $\mathrm{C} 7$ & $\mathrm{C} 8$ & $\mathrm{C} 9$ & $\mathrm{C} 10$ & $\mathrm{C} 11$ & $\mathrm{C} 12$ \\
\hline $\mathrm{u}\left(\mathrm{mm}^{2}\right)$ & 17,26 & 20,69 & 19,23 & 20,13 & 19,67 & 26,13 \\
$\mathrm{k}$ & 0,38 & 0,40 & 0,48 & 0,39 & 0,34 & 0,36 \\
$\mathrm{kp}\left(\mathrm{mm}^{2}\right)$ & 2,87 & 2,90 & 3,31 & 2,87 & 2,87 & 2,87 \\
& 1,08 & 1,17 & 1,58 & 1,11 & 0,98 & 1,05 \\
\hline
\end{tabular}

Observando os resultados da tabela 5, pode ser notado que a incerteza padrão combinada associada à medição da área de reforço dos 12 corpos de prova variou entre 0,36 e $0,52 \mathrm{~mm}^{2}$. Por sua vez, a incerteza expandida apresentou valores de 0,98 até $1,72 \mathrm{~mm}^{2}$, com uma probabilidade de abrangência de 95,45 $\%$. Algumas medidas podem ser tomadas com o intuito de diminuir a incerteza de medição, dentre elas: aumentar o número de leituras de 3 para 5; utilizar um padrão com uma resolução maior durante a calibração do sistema óptico; por fim, efetuar o corte dos corpos de prova de forma muito cuidadosa, para que o desvio de perpendicularidade dos mesmos seja o menor possível.

\section{Conclusões}

Ao finalizar o trabalho, as seguintes conclusões podem ser apresentadas:

a) A incerteza associada à medição da área de reforço foi estimada conforme recomendado pelo ISO GUM. Os valores encontrados variam entre $\pm 0,98 \mathrm{e} \pm 1,72 \mathrm{~mm}^{2}$ para os cordões de solda 1 e 2 , respectivamente, considerando uma probabilidade de abrangência de 95,45\%. De forma similar pode ser estimada a incerteza associada à medição dos demais parâmetros geométricos.

b) Com relação à qualidade dos corpos de prova pode-se dizer que o desvio de planeza dos mesmos apresentou valores pequenos, que não influenciam os resultados das medições. Especial atenção deve ser prestada ao posicionamento e à fixação das placas durante o corte para impedir o aparecimento de desvios de perpendicularidade significativos, pois estes influenciam o resultado das medições e a incerteza final.

\section{Agradecimentos}

Os autores agradecem à FAPEMIG pelo apoio financeiro.

\section{Referências Bibliográficas}

[1] KEJELIN, ZANETTE, N., DE ALMEIDA, BUSCHINELLI, A.J., MEIRELLES, POPE, A. Influence of Welding parameters on the formation of partially diluted zones of dissimilar metal welds. Soldagem \& Inspeção, v. 12, n. 3, p.195-303, jul/sept 2007.

[2] DÍAZ-CEDRÈ, E., CRUZ-CRESPO, A., RAMOS, MORALES, F., TELO, RICO, M., CHAPARRO GONZALEZ, J., RODRIGUEZ, PÉREZ, M., POZO, MOREJÓN, J. Influencia de la relación $\mathrm{O}_{2} / \mathrm{CO}_{2}$ y de la corriente de soldadura sobre la geometría de la unión soldada de bordes rectos en aceros de bajo carbono con el proceso GMAW. Soldagem Insp. Säo Paulo, v. 13, n. 4, p.319-328, Out/Dez 2008.

[3] NAGESH, D. S., DATTA, G. L. Prediction of weld bead geometry and penetration in Shilded Metal-arc Welding using artificial neural networks. Journal of Materials Processing Technology, 123, p. 303-312, 2002.

[4] LÓPEZ IBARRA A. Influencia del coeficiente de dilución sobre la dureza de la capa aportada en el relleno superficial. 5ta Jornada de Investigación. Universidad Autónoma de Zacatecas. 25-29 de junio de 2001.

[5] WANG X. H., ZHANG M. ZOU Z. D., QUO, S. Y. Microestructure and wear properties of TiC-VC reinforced iron based hardfacing layers. Materials Science and Technology, vol 22, No 2, p. 193-198, 2006.

[6] CRUZ-CRESPO, A., SCOTTI,A., PEREZ, M. R. Operacional Behaviour Assessment of Coated Tubular Electrodes for SMAW Hardfacing, J. Mater. Process. Technol., p 265-273, 2007.

[8] KARAOGLU, S., SEgGIN, A. Sensitivity Analysis of Submerged Arc Welding Process Parameters. Journal of Materials Processing Technology, 202, p. 500-507, 2008.

[9] DE OLIVEIRA, RODRIGUES, L., DE PAIVA, A., DA COSTA, S. Otimização do Processo de Soldagem com Eletrodo Tubular através da Análise da Geometria do Cordão de Solda. Soldagem \& Inspeção, v. 13, n. 2, p. 118-127, 2008.

[10] TARNG, Y. S., JUANG, S. C., CHANG, C. H. The use of grey -based Taguchi methods to determine submerged arc welding process-parámetrs in hardfacing. Journal of materials Processing Technology, 128, p. 1-6, 2002.

[11] DEAM, ROMAN, T., BEDNARZ, BERNIE, E., FRANCIS, JOHN A. Welding Parameter, that control dilution in hard facing overlays. Australasian Welding Journal, v. 51, n. 3, p. 41-48, 
2006.

[12] NBR 6409 "Tolerâncias geométricas - Tolerâncias de forma, orientação, posição e batimento - Generalidades, símbolos, definições e indicações em desenho" Maio 1997. p.19. [13] ISO TAG 4/WG 3 "Guide to the Expression of Uncertainty in Measurement", Geneva Switzerland, 1993.

[14] NBR ISO/IEC 17025 "Requisitos gerais para competência de laboratórios de ensaio e calibração". Janeiro 2001.

[15] COMMITTEE OF THE RUSSIAN FEDERATION FOR STANDARDIZATION, Gost 14771-85.

[16] CENTRO DE INVESTIGACIONES DE SOLDADURA. Procedimiento CIS-MET-030: Preparación de muestras, CISUCLV, 2002.

[17] CENTRO DE INVESTIGACIONES DE SOLDADURA. Procedimiento CIS-MET-027: Observación de muestras por microscopia óptica, CIS-UCLV, 2002.

[18] CATTANI AOKI, C. S., GUILHERME DE ARAGÃO, B. J., CORREA, D. O., LIMA, H. T., RAMELLA, T. "Estimativa da Resolução do Microscópio Metalográfico”. ENQUALAB-2008, 09 a 12 de junho de 2008, São Paulo, Brasil. 